\title{
Time Series Forecasting with Interval Type-2 Intuitionistic Fuzzy Logic Systems
}

\author{
Imo Eyoh, Student Member, IEEE ${ }^{1,2}$, Robert John, Senior Member, IEEE ${ }^{1,2}$ and Geert De Maere 2 \\ ${ }^{1}$ Laboratory for Uncertainty in Data and Decision Making (LUCID) \\ ${ }^{2}$ Automated Scheduling, Optimisation and Planning (ASAP) \\ School of Computer Science, University of Nottingham, UK \\ Email: (ije, rij,gdm)@cs.nott.ac.uk
}

\begin{abstract}
Conventional fuzzy time series approaches make use of type-1 or type-2 fuzzy models. Type-1 models with one index (membership grade) cannot fully handle the level of uncertainty inherent in many real world applications. The type-2 models with upper and lower membership functions do handle uncertainties in many applications better than its type- 1 counterparts. This study proposes the use of interval type-2 intuitionistic fuzzy logic system of Takagi-Sugeno-Kang (IT2IFLS-TSK) fuzzy inference that utilises more parameters than type-2 fuzzy models in time series forecasting. The IT2IFLS utilises more indexes namely upper and lower non-membership functions. These additional parameters of IT2IFLS serve to refine the fuzzy relationships obtained from type-2 fuzzy models and ultimately improve the forecasting performance. Evaluation is made on the proposed system using three real world benchmark time series problems namely: Santa Fe, tree ring and Canadian lynx datasets. The empirical analyses show improvements of prediction of IT2IFLS over other approaches on these datasets.
\end{abstract}

\section{INTRODUCTION}

$\mathrm{T}$ ME series forecasting is an important application area that has been extensively researched. It involves the sequential collection of observations over time with the purpose of developing a model that captures the underlying dependencies among attributes of the data. A wide range of approaches have been employed in the analysis of time series data. More recently, the use of soft computing methodologies such as fuzzy logic (type-1 and type-2), neural networks, simulated annealing and genetic algorithms have been reported in the literature for time series forecasting [1]-[4]. These latter approaches have shown significant improvements over the traditional statistical methods because they are non-linear and are able to approximate any complex dynamical systems better than linear statistical models [5].

Due to the prevalent uncertainty in data, fuzzy logic models have been widely adopted. The reason behind this is that the general framework of fuzzy logic connotes uncertainty. However, because fuzzy logic systems lack the learning capability, they are often hybridised with learning algorithms such as artificial neural networks (ANNs) - an approach that is also adopted in this study. Fuzzy set (FS) theory was introduced by Zadeh [6] as a generalisation of the classical notion of a set. From the literature, a type-1 fuzzy logic system (T1FLS) models uncertainty to a certain degree in many applications and may not handle or minimise the effects of uncertainties in some real world applications [7]. Zadeh [8] therefore extended type-1 fuzzy set (T1FS) to include type2 fuzzy set (T2FS) which can handle uncertainties in a better way in many applications because membership grades of T2FS are themselves fuzzy.

Atanassov [9] extended the concept of Zadeh's fuzzy sets to intuitionistic fuzzy sets (IFSs), which handle uncertainty using both the degrees of membership and non-membership of an element $x$ to a fuzzy set $A$ together with some degree of hesitancy. Intuitionistic fuzzy sets of type-1 have been successfully employed in time series forecasting [10], [11]. However, because type-1 intuitionistic fuzzy sets (T1IFS) have membership and non-membership grades that are precise, they may not handle uncertainty well in many applications (see [12]).

To this end, we propose an application of the recently developed interval type-2 intuitionistic fuzzy logic system (IT2IFLS) [12] framework for time series analysis. The IT2IFLS utilises extra degrees of freedom in terms of interval non-membership functions. This constitute a point of departure of our model from other existing classical fuzzy models. Our motivation is to apply IT2IFS to model uncertainty in time series data with the objective of obtaining the minimum prediction error.

The rest of the paper is structured as follows: In section II, IFS, T2IFS and IT2IFS are defined. In section III, IT2IFLS is designed and parameter update rules are derived. We present our results in Section IV, and conclude in section V.

\section{Type-1 And Type-2 Intuitionistic FuZzy Set}

\section{A. Intuitionistic Fuzzy Set (IFS)}

Definition 1. [9] Given $X$ as a non-empty set, an intuitionistic fuzzy set $A^{*}$ in $X$ can be represented as: $A^{*}=$ $\left.\left\{\left(x, \mu_{A^{*}}(x), \nu_{A^{*}}(x)\right): x \in X\right)\right\}$, where $\mu_{A^{*}}(x): X \rightarrow[0,1]$ is the membership grade and $\nu_{A^{*}}(x): X \rightarrow[0,1]$ is the nonmembership grade of element $x \in X$ and for each $x \in X$, $0 \leq \mu_{A^{*}}(x)+\nu_{A^{*}}(x) \leq 1$.

When $\nu_{A^{*}}(x)=1-\mu_{A^{*}}(x)$ for all $x \in X$, then $A$ is a fuzzy set. Moreover, $\pi_{A^{*}}(x)=1-\left(\mu_{A^{*}}(x)+\nu_{A^{*}}(x)\right)$ defines the hesitancy of elements $x$ in $A^{*}$. 


\section{B. Type-2 Intuitionistic Fuzzy Set (T2IFS)}

A T2IFS [12] $\tilde{A}^{*}$ in $X$ consists of type- 2 membership and non-membership grades of $x \in X$ defined as $\mu_{\tilde{A}^{*}}(x, u): u \in$ $J_{x}^{\mu} \subseteq[0,1]$ and $\nu_{\tilde{A}^{*}}(x, u): u \in J_{x}^{\nu} \subseteq[0,1]$ respectively. where $J_{x}^{\mu}$ and $J_{x}^{\nu}$ in the domain $(x, u)$ are defined as follows [12]:

$$
\begin{gathered}
J_{x}^{\mu}=\left\{(x, u): u \in\left[\underline{\mu}_{\tilde{A}^{*}}(x), \bar{\mu}_{\tilde{A}^{*}}(x)\right]\right\} \\
J_{x}^{\nu}=\left\{(x, u): u \in\left[\underline{\nu}_{\tilde{A}^{*}}(x), \bar{\nu}_{\tilde{A}^{*}}(x)\right]\right\}
\end{gathered}
$$

for membership and non-membership functions respectively.

Definition 2. A T2IFS [12] denoted by $\tilde{A}^{*}$ has a type-2 membership function $\mu_{\tilde{A}^{*}}(x, u)$, and a type-2 non-membership function $\nu_{\tilde{A}^{*}}(x, u)$, i.e.,

$$
\begin{aligned}
\tilde{A}^{*}=\left\{(x, u), \mu_{\tilde{A^{*}}}(x, u), \nu_{\tilde{A}^{*}}(x, u) \mid\right. & \forall x \in X, \\
& \left.\forall u \in J_{x}^{\mu}, \forall u \in J_{x}^{\nu}\right\}
\end{aligned}
$$

in which $0 \leq \mu_{\tilde{A}^{*}}(x, u) \leq 1$ and $0 \leq \nu_{\tilde{A}^{*}}(x, u) \leq 1$ where $\forall u \in J_{x}^{\mu}$ and $\forall u \in J_{x}^{\nu}$ conform to the T1 constraint that $0 \leq \mu_{A^{*}}(x)+\nu_{A^{*}}(x) \leq 1$. Thus, when uncertainties disappear, a type-2 membership and non-membership functions must collapse to a type-1 membership and nonmembership functions respectively and their amplitudes must lie in the interval $[0,1]$. That is, $0 \leq \mu_{\tilde{A}^{*}}(x, u) \leq 1$ and $0 \leq \nu_{\tilde{A}^{*}}(x, u) \leq 1$. Alternatively,

$$
\int_{x \in X}\left[\int_{u \in J_{x}^{\mu}} \int_{u \in J_{x}^{\nu}}\left\{\mu_{\tilde{A}^{*}}(x, u), \nu_{\tilde{A}^{*}}(x, u)\right\}\right] /(x, u)
$$

where $\iiint$ represents union over all admissible values of $x$ and $u$ for a continuous universe of discourse, and $\sum$ is used instead for discrete universe of discourse. When $\mu_{\tilde{A}^{*}}(x, u)=1$ and $\nu_{\tilde{A}^{*}}(x, u)=1$, a simpler version (IT2IFS) of a T2IFS is obtained. (see Figure 1 and Equation (1)).

Definition 3. [13] An IT2IFS, $\tilde{A}^{*}$, consists of membership and non-membership bounding functions defined as $\bar{\mu}_{\tilde{A}^{*}}(x)$, $\underline{\mu}_{\tilde{A}^{*}}(x)$ and $\bar{\nu}_{\tilde{A}^{*}}(x), \underline{\nu}_{\tilde{A}^{*}}(x)$ respectively for all $x \in X$ with constraints: $0 \leq \bar{\mu}_{\tilde{A}^{*}}(x)+\underline{\nu}_{\tilde{A}^{*}}(x) \leq 1$ and $0 \leq \underline{\mu}_{\tilde{A}^{*}}(x)+$ $\bar{\nu}_{\tilde{A}^{*}}(x) \leq 1$.

The IT2IFLS utilises two IF-indices which are defined as follows [12]:

$$
\begin{gathered}
\pi_{c}(x)=\max \left(0,\left(1-\left(\mu_{\tilde{A}^{*}}(x)+\nu_{\tilde{A^{*}}}(x)\right)\right)\right) \\
\bar{\pi}_{v a r}(x)=\max \left(0,\left(1-\left(\bar{\mu}_{\tilde{A}^{*}}(x)+\underline{\nu}_{\tilde{A}^{*}}(x)\right)\right)\right) \\
\underline{\pi}_{v a r}(x)=\max \left(0,\left(1-\left(\underline{\mu}_{\tilde{A}^{*}}(x)+\bar{\nu}_{\tilde{A}^{*}}(x)\right)\right)\right)
\end{gathered}
$$

such that: $0 \leq \pi_{c}(x) \leq 1$ and $0 \leq \pi_{v a r}(x) \leq 1$.

As defined above, an IT2IFS $\tilde{A}^{*}$ is made up of interval type2 membership function, $\mu_{\tilde{A}^{*}}(x, u)$ and interval type- 2 nonmembership function, $\nu_{\tilde{A}^{*}}(x, u)$ for every $x \in X$ expressed as in Eqn (1) [12].

$$
\begin{aligned}
& \tilde{A}^{*}=\int_{x \in X} \int_{u \in J_{x}^{\mu}} \int_{u \epsilon J_{x}^{\nu}} 1 /(x, u) \\
& =\int_{x \in X}\left[\int_{u \in J_{x}^{\mu}} \int_{u \in J_{x}^{\nu}} 1 /(u)\right] / x
\end{aligned}
$$

where $x$ is the primary variable, and $u$ is the secondary variable. The uncertainty about an IT2IFS is completely described by membership and non-membership footprints of uncertainty (FOUs) (see Figure (1)) and defined as in Eqns (2) and (3) [12]

$$
\begin{aligned}
& \operatorname{FOU}_{\mu}\left(\tilde{A}^{*}\right)=\bigcup_{\forall x \in X}\left[\underline{\mu}_{\tilde{A}^{*}}(x), \bar{\mu}_{\tilde{A}^{*}}(x)\right] \\
& \operatorname{FOU}_{\nu}\left(\tilde{A}^{*}\right)=\bigcup_{\forall x \in X}\left[\underline{\nu}_{\tilde{A}^{*}}(x), \bar{\nu}_{\tilde{A}^{*}}(x)\right]
\end{aligned}
$$

\section{InTERVAL TyPe-2 INTUITIONISTIC FUZZy LOGIC SYSTEM}

Similar to a type-2 fuzzy logic system (T2FLS), a T2IFLS consists of the fuzzifier, rule base, fuzzy inference engine and output processing module.

\section{A. Fuzzification}

The fuzzification process employed is the interval singleton type-2 fuzzification and involves the mapping of a numeric input vector $\mathbf{x} \in X$ into an IT2IFS $\tilde{A}^{*}$ in $\mathrm{X}$ which activates the inference engine. Here, interval singleton type-2 fuzzification is employed. Hence, for every input $x=x^{\prime}$, crisp numerical values are obtained for membership (lower and upper values) and non-membership (lower and upper values) with value 0 in all other positions where $x \neq x^{\prime}$. The firing strength for membership function and non-membership functions are intervals $\left[\underline{f^{\mu}}, \overline{f^{\mu}}\right]$ and $\left[\underline{f^{\nu}}, \overline{f^{\nu}}\right]$ respectively.

Intuitionistic Gaussian membership and non-membership functions with uncertain standard deviation are utilised for the time series analysis which are defined as follows [12]:

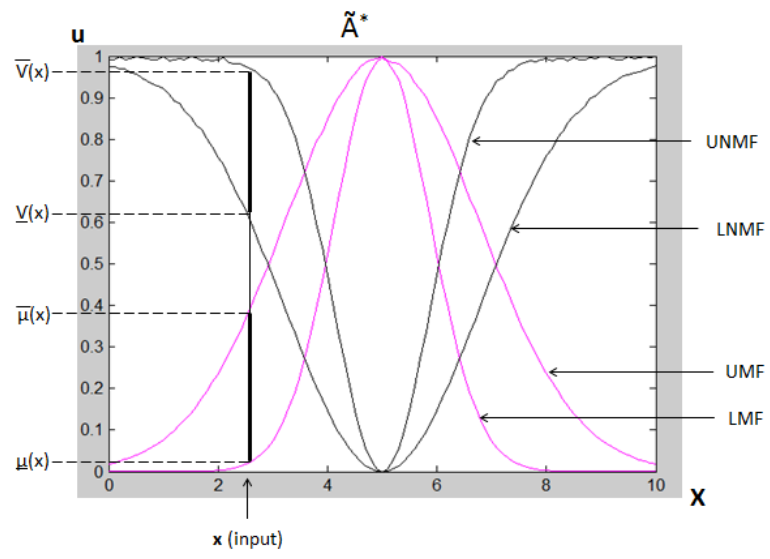

Fig. 1: An IT2 intuitionistic Gaussian membership and nonmembership functions - IT2IFS [12] 


$$
\begin{aligned}
& \overline{\mu_{i k}}\left(x_{i}\right)=\exp \left(-\frac{\left(x_{i}-c_{i k}\right)^{2}}{2 \bar{\sigma}_{2, i k}^{2}}\right) *\left(1-\pi_{c, i k}\left(x_{i}\right)\right) \\
& \underline{\mu_{i k}}\left(x_{i}\right)=\exp \left(-\frac{\left(x_{i}-c_{i k}\right)^{2}}{2 \underline{\sigma}_{1, i k}^{2}}\right) *\left(1-\pi_{c, i k}\left(x_{i}\right)\right) \\
& \overline{\nu_{i k}}\left(x_{i}\right)=\left(1-\bar{\pi}_{v a r, i k}\left(x_{i}\right)\right)-\left[\exp \left(-\frac{\left(x_{i}-c_{i k}\right)^{2}}{2 \bar{\sigma}_{1, i k}^{2}}\right)\right. \\
& \text { * } \left.\left(1-\pi_{c, i k}\left(x_{i}\right)\right)\right] \\
& \underline{\nu_{i k}}\left(x_{i}\right)=\left(1-\underline{\pi}_{v a r, i k}\left(x_{i}\right)\right)-\left[\exp \left(-\frac{\left(x_{i}-c_{i k}\right)^{2}}{2 \underline{\sigma}_{2, i k}^{2}}\right)\right. \\
& \text { * } \left.\left(1-\pi_{c, i k}\left(x_{i}\right)\right)\right]
\end{aligned}
$$

where $\pi_{c, i k}$ is the IF-index of center and $\pi_{v a r, i k}$ is the IFindex of variance [14]. The antecedent parameters are $\bar{\sigma}_{2, i k}$, $\underline{\sigma}_{1, i k}, \pi_{c, i k}$ and $\pi_{v a r, i k}$.

\section{B. Rules}

The IF-THEN rule of an IT2IFLS has the same syntax as that of the classical IT2FLS. The only difference is that IT2IFS are used in the rule formation instead of ordinary IT2FS. The rule representatipn is as follows:

$$
\begin{gathered}
R_{k}: I F x_{1} \text { is } \tilde{A}^{*}{ }_{1 k} \text { and } x_{2} \text { is } \tilde{A}^{*}{ }_{2 k} \text { and } \cdots \text { and } x_{n} \text { is } \tilde{A}^{*}{ }_{n k} \\
\text { THEN } y_{k} \text { is } f\left(x_{1}, x_{2}, \cdots, x_{n}\right) \\
=w_{1 k} x_{1}+w_{2 k} x_{2}+\cdots+w_{n k} x_{n}+b_{k}
\end{gathered}
$$

where $\tilde{A}^{*}{ }_{1 k}, \tilde{A}^{*}{ }_{2 k}, \cdots, \tilde{A}^{*}{ }_{i k}, \cdots, \tilde{A}^{*}{ }_{n k}$ are IT2IFS and $y_{k}$ is the output of the $k t h$ rule formed by linear combination of the input vector: $\left(x_{1}, x_{2}, \cdots, x_{n}\right)$.

\section{Inference}

The IT2IFLS proposed in this study benefits from type-2 intuitionistic membership and non-membership functions in the antecedent parts and a linear function in the consequent parts, otherwise referred to as A2-C0 intuitionistic fuzzy system

The output of IT2IFLS is computed as follows [12]:

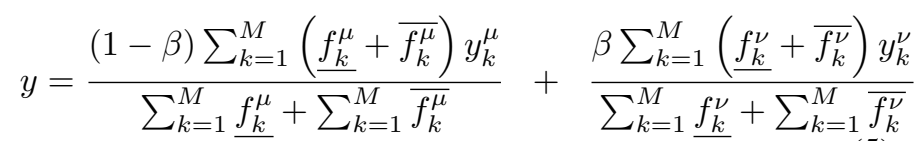

where $f_{k}^{\mu}, \bar{f}_{k}^{\mu}, f_{k}^{\nu}$ and $\bar{f}_{k}^{\nu}$ are the lower membership, upper membership, lower non-membership and upper nonmembership firing strength respectively. The implication operator adopted for the study is the "prod" t-norm such that:

$$
\begin{aligned}
& \underline{f_{k}^{\mu}}(x)=\underline{\mu}_{\tilde{A}^{*}{ }_{1 k}}\left(x_{1}\right) * \underline{\mu}_{\tilde{A}^{*}{ }_{2 k}}\left(x_{2}\right) * \cdots * \underline{\mu}_{\tilde{A}^{*}{ }_{n k}}\left(x_{n}\right) \\
& \overline{f_{k}^{\mu}}(x)=\bar{\mu}_{\tilde{A}^{*}{ }_{1 k}}\left(x_{1}\right) * \bar{\mu}_{\tilde{A}^{*}{ }_{2 k}}\left(x_{2}\right) * \cdots * \bar{\mu}_{\tilde{A}^{*}{ }_{n k}}\left(x_{n}\right) \\
& \underline{f_{k}^{\nu}}(x)=\underline{\nu}_{\tilde{A}^{*}{ }_{1 k}}\left(x_{1}\right) * \underline{\nu}_{\tilde{A}^{*}{ }_{2 k}}\left(x_{2}\right) * \cdots * \underline{\nu}_{\tilde{A}^{*}{ }_{n k}}\left(x_{n}\right) \\
& \overline{f_{k}^{\nu}}(x)=\bar{\nu}_{\tilde{A}^{*}{ }_{1 k}}\left(x_{1}\right) * \bar{\nu}_{\tilde{A}^{*}{ }_{2 k}}\left(x_{2}\right) * \cdots * \bar{\nu}_{\tilde{A}^{*}{ }_{n k}}\left(x_{n}\right)
\end{aligned}
$$

where $*$ is the "prod" operator, $y_{k}^{\mu}$ and $y_{k}^{\nu}$ are the output of the $k t h$ rule corresponding to membership and non-membership functions respectively. The user defined parameter $\beta$ specifies the contribution of the membership and non-membership values in the final output, where $\beta \in[0,1]$. It follows that if $\beta=0$, only the membership function is involved in the output generation and if $\beta=1$, then the system's outputs is a function of only the non-membership. The parameters of the antecedent and consequent parts of the rules are tuned using gradient descent algorithm (GDA). The cost function for a single output is defined as follows:

$$
E=\frac{1}{2}\left(y^{a}-y\right)^{2}
$$

where $y^{a}$ is the actual output and $y$ is the simulated output. The parameter update rules are as follows:

$$
\begin{aligned}
w_{i k}(t+1) & =w_{i k}(t)-\gamma \frac{\partial E}{\partial w_{i k}} \\
b_{k}(t+1) & =b_{k}(t)-\gamma \frac{\partial E}{\partial b_{k}} \\
c_{i k}(t+1) & =c_{i k}(t)-\gamma \frac{\partial E}{\partial c_{i k}} \\
\sigma_{1, i k}(t+1) & =\sigma_{1, i k}(t)-\gamma \frac{\partial E}{\partial \sigma_{1, i k}} \\
\sigma_{2, i k}(t+1) & =\sigma_{2, i k}(t)-\gamma \frac{\partial E}{\partial \sigma_{2, i k}}
\end{aligned}
$$

where $\gamma$ is the learning rate(step size). The derivatives in Equations (6) and (7) are computed as follows:

$$
\frac{\partial E}{\partial w_{i k}}=\frac{\partial E}{\partial y} \frac{\partial y}{\partial y_{k}} \frac{\partial y_{k}}{\partial w_{i k}}=\sum_{k} \frac{\partial E}{\partial y}\left[\frac{\partial y}{\partial y_{k}^{\mu}} \frac{\partial y_{k}^{\mu}}{\partial w_{i k}^{\mu}}+\frac{\partial y}{\partial y_{k}^{\nu}} \frac{\partial y_{k}^{\nu}}{\partial w_{i k}^{\nu}}\right]
$$

where $y_{k}$ is defined as in Equation (4). The derivatives in Equations (8) to (10) are computed as follows:

$$
\begin{aligned}
& \frac{\partial E}{c_{i k}}=\sum_{k} \frac{\partial E}{\partial y}[ \frac{\partial y}{\partial \underline{f}_{k}^{\mu}} \frac{\partial \underline{f}_{k}^{\mu}}{\partial \underline{\mu}_{i k}} \frac{\partial \underline{\mu}_{i k}}{\partial c_{i k}}+\frac{\partial y}{\partial \bar{f}_{k}^{\mu}} \frac{\partial \bar{f}_{k}^{\mu}}{\partial \bar{\mu}_{i k}} \frac{\partial \bar{\mu}_{i k}}{\partial c_{i k}} \\
&+\left.\frac{\partial y}{\partial \underline{f}_{k}^{\nu}} \frac{\partial \underline{f}_{k}^{\nu}}{\partial \underline{\nu}_{i k}} \frac{\partial \underline{\nu}_{i k}}{\partial c_{i k}}+\frac{\partial y}{\partial \bar{f}_{k}^{\nu}} \frac{\partial \bar{f}_{k}^{\nu}}{\partial \bar{\nu}_{i k}} \frac{\partial \bar{\nu}_{i k}}{\partial c_{i k}}\right] \\
& \frac{\partial E}{\sigma_{1, i k}}=\sum_{k} \frac{\partial E}{\partial y}\left[\frac{\partial y}{\partial \underline{f}_{k}^{\mu}} \frac{\partial \underline{f}_{k}^{\mu}}{\partial \underline{\mu}_{i k}} \frac{\partial \underline{\mu}_{i k}}{\partial \sigma_{1, i k}^{\mu}}+\frac{\partial y}{\partial \underline{f}_{k}^{\nu}} \frac{\partial \underline{f}_{k}^{\nu}}{\partial \underline{\nu}_{i k}} \frac{\partial \underline{\nu}_{i k}}{\partial \sigma_{2, i k}^{\nu}}\right] \\
& \frac{\partial E}{\sigma_{2, i k}}=\sum_{k} \frac{\partial E}{\partial y}\left[\frac{\partial y}{\partial \bar{f}_{k}^{\mu}} \frac{\partial \bar{f}_{k}^{\mu}}{\partial \bar{\mu}_{i k}} \frac{\partial \bar{\mu}_{i k}}{\partial \sigma_{2, i k}^{\mu}}+\frac{\partial y}{\partial \bar{f}_{k}^{\nu}} \frac{\partial \bar{f}_{k}^{\nu}}{\partial \bar{\nu}_{i k}} \frac{\partial \bar{\nu}_{i k}}{\partial \sigma_{1, i k}^{\nu}}\right]
\end{aligned}
$$

In the next section, we carry out the evaluation of the developed model to assess its efficiency and performance on three real world application domains.

\section{EXPERIMENTS AND RESULTS}

In this section, three real world applications are investigated to verify the feasibility and effectiveness of the proposed 
IT2IFLS on time series datasets namely Santa Fe, tree ring and Canadian lynx time series. The experiments are conducted and the results are compared with similar studies in the literature. The performance criteria used for the experiments are the root mean square error (RMSE), the non-dimensional error index (NDEI) and the mean absolute error (MAE) as expressed in Equations (11) to (13) respectively.

$$
\begin{gathered}
R M S E=\sqrt{\frac{1}{N} \sum_{i=1}^{N}\left(y^{a}-y\right)^{2}} \\
N D E I=\frac{R M S E}{\operatorname{std}\left(y^{a}\right)} \\
M A E=\frac{1}{N} \sum_{i=1}^{N}\left|y^{a}-y\right|
\end{gathered}
$$

where $y^{a}$ is the desired output and $y$ is the output of the model, $N$ is the number of testing data points.

Two intuitionistic Gaussian membership and nonmembership functions are utilised for all experiments. The value of $\beta$ is initially set to 0.5 to allow equal initial contribution of membership and non-membership functions to the model outputs. The values for the weights $(w)$ and bias $(b)$ are randomly generated in the interval $[0,1]$. All experiments are carried out using $M A T L A B^{\circledR} 2014$ running on a 64-bit Intel core i3-4130 CPU@3.40GHz /8GB RAM configuration computer.

\section{A. Example 1 - Santa Fe time series}

This example considers the Santa Fe Laser dataset of the Santa Fe time series competition obtained from [15]. The data were measured from a far-infra-red laser in a chaotic state. This series was earlier analysed in [16], and a model called pattern modeling and recognition system (PMRS) was proposed. The performance comparison using neural network (NN) and a statistical exponential-smoothing (ES) was also reported. The Santa Fe time series is a univariate time series measured from a physical system in the laboratory. The computational protocols are similar to [17], 1000 samples are used with five inputs and one output with the data generation vector represented as: $y(t)=(y(t-1) y(t-2) y(t-3) y(t-4) y(t-5))$. All samples are scaled to within the range $[0,1]$ by dividing each by the maximum value of the dataset. Similar to [17], $90 \%$ of the samples are used for training while $10 \%$ are used for testing. The parameters $\beta=\alpha=0.5$ with 100 training epochs. Table I shows the performance of IT2IFLS with other fuzzy approaches on both the training and test set. The results show that IT2IFLS reduces the RMSE of the test set compared to the non-fuzzy and other fuzzy approaches except SVR-FM with $\epsilon=0.001$. The reason for this could be in the large number of parameters (4484 parameters) which could lead to the possible improvement in the approximation capability of SVR-FM $(\epsilon=0.001)$. The performance of IT2IFLS on the test set of Santa-Fe time series is an indication of a good generalisation capability of the model.

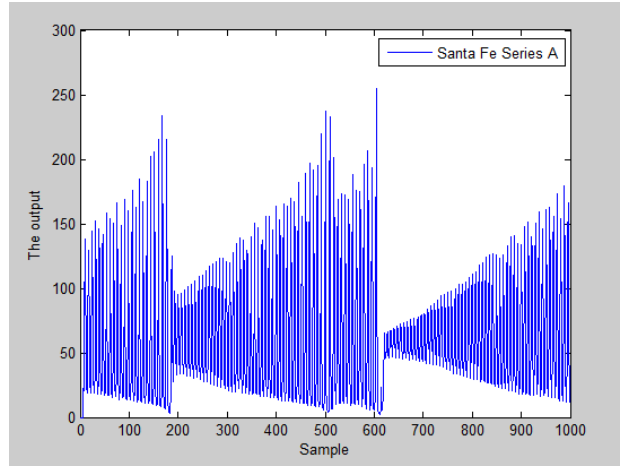

Fig. 2: Plot of Santa Fe laser time series dataset

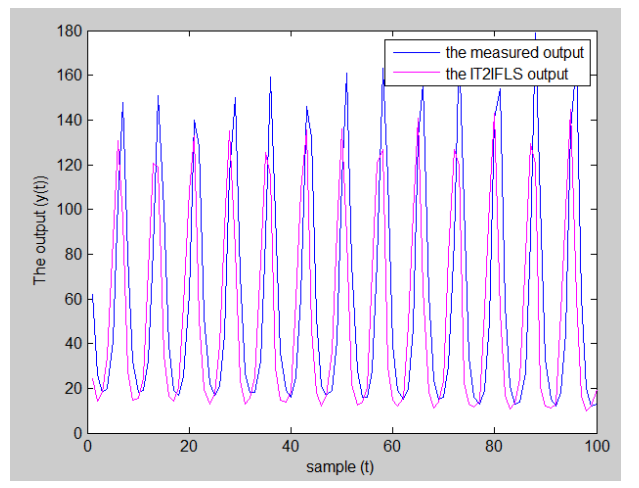

Fig. 3: IT2IFLS prediction output of Santa Fe Laser

\section{B. Tree Ring Time Series}

The tree ring time series obtained from [21] contains annual measures of tree rings width measured in Argentina for the period 441-1974. The dataset is randomly split into $75 \%$ training and $25 \%$ testing. In [22], the evolving fuzzy optimally pruned extreme learning machine (eF-OP-ELM), was reported for analysing this time series. The dynamic evolving neuro-fuzzy inference system (DENFIS), evolving Takagi-Sugeno (eTS) model and online sequential method for fuzzy systems based on online sequential ELM (OS-fuzzy-ELM) were also reported in [22] for tree ring time series analysis. All computational protocols in this study are arranged as close as possible to those reported in [22] to ease comparison. Table II shows the average cross validation NDEI and standard deviation (Std) of the tree ring dataset for 25 trials. From Table II, IT2IFLS outperforms other fuzzy models with reduced NDEI.

TABLE II: Tree ring time series forecasting

\begin{tabular}{|c|c|c|}
\hline Models & NDEI & Std NDE \\
\hline DENFIS [23] & 0.959 & 0.624 \\
eTS [24] & 0.714 & 0.457 \\
OS-Fuzzy-ELM [25] & 0.794 & 0.511 \\
eF-OP-ELM [22] & 0.841 & 0.536 \\
IT2IFLS-TSK & 0.395 & 0.157 \\
\hline
\end{tabular}

\section{Canadian Lynx Time series}

This time series is selected in order to compare the performance of IT2IFLS on non-fuzzy approaches. Canadian lynx 
TABLE I: Performance comparison of IT2IFLS with other models on Santa Fe time series A dataset

\begin{tabular}{|c|c|c|c|c|}
\hline Models & Rule Number & Parameter number & Training RMSE & Test RMSE \\
\hline ES [16] & - & - & - & 56.20 \\
\hline NN [16] & - & - & - & 24.6 \\
\hline PMRS [16] & - & - & - & 14.23 \\
\hline SONFIN [18] & 9 & 144 & 6.956 & 5.983 \\
\hline T2FLS-G & 5 & 135 & 8.50 & 7.16 \\
\hline SEIT2FNN [19] & 5 & 135 & 7.677 & 5.766 \\
\hline IT2FNN-SVR(N) [17] & 5 & 106 & 13.565 & 4.337 \\
\hline IT2FNN-SVR(F) [17] & 5 & 106 & 9.094 & 3.474 \\
\hline SVR-FM $(\epsilon=0.1)[20]$ & 31 & 188 & 14.370 & 9.707 \\
\hline SVR-FM $(\epsilon=0.001)[20]$ & 747 & 4484 & 7.069 & 1.650 \\
\hline IT2IFLS-TSK & 32 & 434 & 8.355 & 2.261 \\
\hline
\end{tabular}

dataset is a time series that shows the number of lynx trapped in the Mckenzie river district per year in northern Canada and corresponds to the period 1821-1934. Similar to previous studies such as [26]-[28], the logarithms to the base 10 of the data are used in the analysis. Figures 4 and 5 show the original and the logarithmic transformed data of the Canadian lynx series respectively, with a periodicity of approximately 10 years. The series consists of 114 observations of which 100 samples are used for training and the remaining 14 are used for testing in order to validate the effectiveness of the model proposed in this study. Similar to [28], the maximum training epoch adopted is 2000. As shown in Table III, IT2IFLS outperforms the listed non-fuzzy approaches on the Canadian lynx dataset.

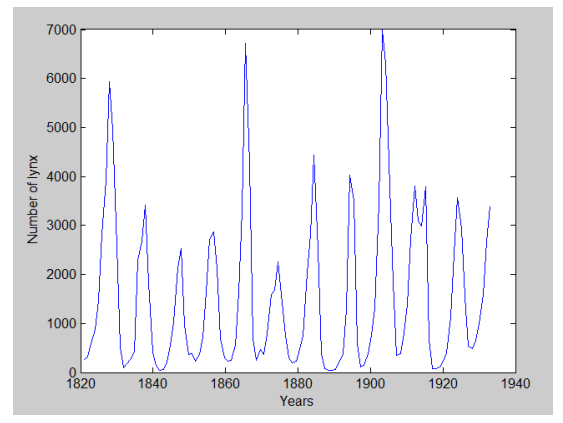

Fig. 4: Original Canadian lynx time series data (1821-1934)

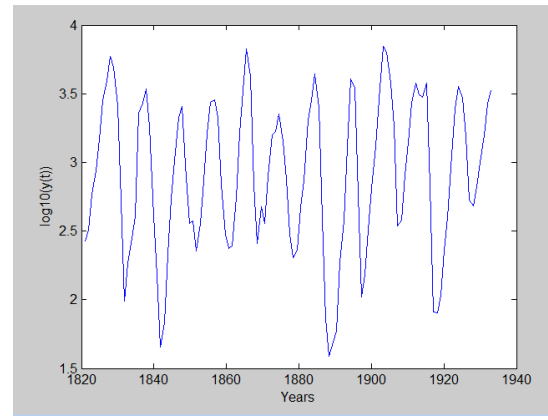

Fig. 5: Transformed Canadian lynx time series data
TABLE III: Performance comparison of IT2IFLS with nonfuzzy models on Canadian lynx time series

\begin{tabular}{|c|c|c|}
\hline Models & MSE & MAE \\
\hline Zhang's ARIMA [26] & 0.020486 & 0.112255 \\
\hline ANN [26] & 0.020466 & 0.112109 \\
\hline ANN (p,d,q) [29] & 0.013609 & 0.089625 \\
\hline Zhang's Hybrid & & \\
ARIMA/ANNs model [26] & 0.017233 & 0.103972 \\
\hline Hybrid ARIMA/ERNN model [30] & 0.009 & - \\
\hline SETAR [31] & 0.014 & - \\
\hline FNN [31] & 0.009 & - \\
\hline Generalised Hybrid & & \\
ARIMA/ANNs model [32] & 0.00999 & 0.085055 \\
\hline ANN/PNN model [27] & 0.014872 & 0.079628 \\
\hline ARIMA/PNN model [27] & 0.011461 & 0.084381 \\
\hline MNM-ANN-DEA [33] & 0.00663 & - \\
\hline GA-BPNN & 0.013599 & 0.081477 \\
\hline DE-BPNN & 0.012899 & 0.080542 \\
\hline ANN Ensemble & 0.00715 & - \\
\hline RBF-AR & 0.0073 & - \\
\hline ADE-BPNN [28] & 0.010392 & 0.070723 \\
\hline GLSSVM & 0.00560 & 0.0552 \\
\hline L\&NL-ANN & 0.006 & - \\
\hline IT2IFLS-TSK & 0.00463 & 0.0205 \\
\hline & \multicolumn{2}{|c}{} \\
\hline &
\end{tabular}

\section{CONClusion}

In this study, an IT2IFLS-TSK approach to time series forecasting is presented. The IT2IFLS can accommodate more imprecision in terms of non-membership function, thereby modelling imperfect and imprecise knowledge better than some classical fuzzy approaches. The key contribution in this study is the integration of non-membership function and IF-index in IT2FLS (IT2IFLS). These extra parameters of IT2IFLS give it additional design degrees of freedom to handle uncertainties well. In future, we intend to learn the parameters of the IT2IFLS using hybrid approach of GD and Kalman filter algorithms. We also intend to apply IFS to general T2FLS.

\section{ACKNOWLEDGEMENT}

This research work was funded by the Government of Nigeria under the Tertiary Education Trust Fund (TETFund).

\section{REFERENCES}

[1] I. Rojas, O. Valenzuela, F. Rojas, A. Guillén, L. J. Herrera, H. Pomares, L. Marquez, and M. Pasadas, "Soft-computing techniques and arma 
model for time series prediction," Neurocomputing, vol. 71, no. 4, pp. 519-537, 2008.

[2] Y. Chen, B. Yang, and J. Dong, "Time-series prediction using a local linear wavelet neural network," Neurocomputing, vol. 69, no. 4, pp. 449465, 2006.

[3] G. G. Szpiro, "Forecasting chaotic time series with genetic algorithms," Physical Review E, vol. 55, no. 3, p. 2557, 1997.

[4] M. Almaraashi and R. John, "Tuning of type-2 fuzzy systems by simulated annealing to predict time series," in Proceedings of the World Congress on Engineering, vol. 2, 2011, pp. 976-980.

[5] O. Castillo and P. Melin, "Comparison of hybrid intelligent systems, neural networks and interval type-2 fuzzy logic for time series prediction," in 2007 International Joint Conference on Neural Networks. IEEE, 2007, pp. 3086-3091.

[6] L. A. Zadeh, "Fuzzy sets," Information and control, vol. 8, no. 3, pp. 338-353, 1965.

[7] J. M. Mendel, "Uncertain rule-based fuzzy logic system: introduction and new directions," 2001.

[8] L. A. Zadeh, "The concept of a linguistic variable and its application to approximate reasoning-i," Information Sciences, vol. 8, pp. 199-249, 1975.

[9] K. T. Atanassov, "Intuitionistic fuzzy sets," Fuzzy sets and Systems, vol. 20, no. 1, pp. 87-96, 1986.

[10] V. Olej and P. Hájek, "If-inference systems design for prediction of ozone time series: the case of pardubice micro-region," in Artificial Neural Networks-ICANN 2010. Springer, 2010, pp. 1-11.

[11] O. Castillo, A. Alanis, M. Garcia, and H. Arias, "An intuitionistic fuzzy system for time series analysis in plant monitoring and diagnosis," Applied Soft Computing, vol. 7, no. 4, pp. 1227-1233, 2007.

[12] I. Eyoh, R. John, and G. De Maere, "Interval type-2 intuitionistic fuzzy logic system for non-linear system prediction," in 2016 IEEE International Conference on Systems, Man and Cybernetics, 2016, pp. 1063-1068.

[13] D. D. Nguyen, L. T. Ngo, and L. T. Pham, "Interval type-2 fuzzy cmeans clustering using intuitionistic fuzzy sets," in IEEE Third World Congress on Information and Communication Technologies (WICT), 2013, pp. 299-304.

[14] P. Hájek and V. Olej, "Intuitionistic fuzzy neural network: The case of credit scoring using text information," in Engineering Applications of Neural Networks. Springer, 2015, pp. 337-346.

[15] http://www-psych.stanford.edu/ andreas/Time-Series/SantaFe.html, accessed: 2016-07-21

[16] S. Singh, "Noise impact on time-series forecasting using an intelligent pattern matching technique," Pattern Recognition, vol. 32, no. 8, pp. 1389-1398, 1999.

[17] C.-F. Juang, R.-B. Huang, and W.-Y. Cheng, "An interval type-2 fuzzyneural network with support-vector regression for noisy regression problems," IEEE Transactions on Fuzzy Systems, vol. 18, no. 4, pp. 686-699, 2010.

[18] C.-F. Juang and C.-T. Lin, "An online self-constructing neural fuzzy inference network and its applications," IEEE Transactions on Fuzzy Systems, vol. 6, no. 1, pp. 12-32, 1998.

[19] C.-F. Juang and Y.-W. Tsao, "A self-evolving interval type-2 fuzzy neural network with online structure and parameter learning," IEEE Transactions on Fuzzy Systems, vol. 16, no. 6, pp. 1411-1424, 2008.

[20] J.-H. Chiang and P.-Y. Hao, "Support vector learning mechanism for fuzzy rule-based modeling: a new approach," IEEE Transactions on Fuzzy Systems, vol. 12, no. 1, pp. 1-12, 2004.

[21] Hyndman, R. J. Time Series Data Library. http://data.is/TSDLdemo., accessed: 2016-07-30.

[22] F. M. Pouzols and A. Lendasse, "Evolving fuzzy optimally pruned extreme learning machine for regression problems," Evolving Systems, vol. 1, no. 1, pp. 43-58, 2010.

[23] N. K. Kasabov and Q. Song, "Denfis: dynamic evolving neural-fuzzy inference system and its application for time-series prediction," IEEE transactions on Fuzzy Systems, vol. 10, no. 2, pp. 144-154, 2002.

[24] P. P. Angelov and D. P. Filev, "An approach to online identification of takagi-sugeno fuzzy models," IEEE Transactions on Systems, Man, and Cybernetics, Part B (Cybernetics), vol. 34, no. 1, pp. 484-498, 2004.

[25] H.-J. Rong, G.-B. Huang, N. Sundararajan, and P. Saratchandran, "Online sequential fuzzy extreme learning machine for function approximation and classification problems," IEEE Transactions on Systems, Man, and Cybernetics, Part B (Cybernetics), vol. 39, no. 4, pp. 10671072, 2009.
[26] G. P. Zhang, "Time series forecasting using a hybrid arima and neural network model," Neurocomputing, vol. 50, pp. 159-175, 2003.

[27] M. Khashei and M. Bijari, "A new class of hybrid models for time series forecasting," Expert Systems with Applications, vol. 39, no. 4, pp. 4344-4357, 2012.

[28] L. Wang, Y. Zeng, and T. Chen, "Back propagation neural network with adaptive differential evolution algorithm for time series forecasting," Expert Systems with Applications, vol. 42, no. 2, pp. 855-863, 2015.

[29] M. Khashei and M. Bijari, "An artificial neural network (p, d, q) model for timeseries forecasting," Expert Systems with applications, vol. 37, no. 1, pp. 479-489, 2010.

[30] C. H. Aladag, E. Egrioglu, and C. Kadilar, "Forecasting nonlinear time series with a hybrid methodology," Applied Mathematics Letters, vol. 22 , no. 9, pp. 1467-1470, 2009.

[31] Y. Kajitani, K. W. Hipel, and A. I. McLeod, "Forecasting nonlinear time series with feed-forward neural networks: a case study of canadian lynx data," Journal of Forecasting, vol. 24, no. 2, pp. 105-117, 2005.

[32] M. Khashei and M. Bijari, "A novel hybridization of artificial neural networks and arima models for time series forecasting," Applied Soft Computing, vol. 11, no. 2, pp. 2664-2675, 2011.

[33] E. Bas, "The training of multiplicative neuron model based artificial neural networks with differential evolution algorithm for forecasting," Journal of Artificial Intelligence and Soft Computing Research, vol. 6, no. 1 , pp. 5-11, 2016. 